\title{
Opinión de Escolares de una Institución Educativa sobre la Presencia de Violencia en los Medios de Comunicación
}

\section{State school children's opinions regarding violence in the media}

Laura E. Piedrahita S

Escuela de Enfermería. Facultad de Salud. Universidad del Valle. Cali, Colombia. lauraeps1@ hotmail.com

Recibido 2 Septiembre 2008/Enviado para Modificación 23 Marzo 2009/Aceptado 5 Mayo 2009

\section{RESUMEN}

Objetivos Describir la opinión que tienen sobre la violencia percibida a través de los medios de comunicación los escolares de una institución educativa.

Materiales y Métodos Investigación de tipo descriptivo-exploratorio, para la cual se seleccionaron mediante muestreo aleatorio estratificado, 60 niños de una institución educativa del sector oficial de la ciudad de Cali. Se tomaron datos cuantitativos Resultados Se encontraron aspectos comunes relacionados con la opinión sobre la presencia de violencia a través de los medios como son: exposición frecuente a la violencia a través de ellos y además se identificó falta de supervisión por parte de los padres.

Conclusiones El contexto social, así como la observación constante de violencia a través de los medios de comunicación, influyen en la opinión que los escolares tienen sobre el fenómeno de la violencia, así como en su predisposición a ella.

Palabras Clave: Violencia, medios de comunicación, escolares. (fuente: DeCS, BIREME).

\section{ABSTRACT}

Objective To describe the opinion of schoolage children, from a public school, regarding the violence they perceive in the media.

Material and Methods Descriptive-exploratory research with a sample, selected according to the child's age in a public school. Quantitative data were collected.

Results There were found common issues related to the child's opinion about the violence present in the media such as frequent exposure to the media violence and lack of parental supervision.

Conclusions Social context and constant exposure to the media violence affect the children's opinion about the violence phenomenon and their predisposition to it.

Key Words: Violence, communications media, school child (source: MeSH, NLM). 
$\mathrm{S}$ e entiende por violencia "...todas aquellas actuaciones de individuos o grupos que ocasionen la muerte de otros o lesionen a su integridad física o moral". En sentido muy general, la violencia se puede ver como algo que impide la realización de los Derechos Humanos, comenzando por el más fundamental: el derecho a la vida" (1). Como fenómeno, día a día, la violencia se introduce más en nuestra cotidianidad, en el ambiente familiar, social y en el colegio. Los estudios demuestran que cada vez los niños son las personas más afectadas por este fenómeno.

Con el fin de contribuir a la búsqueda de soluciones, consideramos necesario el conocimiento de aquellos factores que inciden en la aparición de la violencia, como son los medios de comunicación y la forma cómo éstos influyen en la percepción que tienen los niños de este fenómeno. Partir de la forma comolos niños comprenden la violencia nos permitirá conocer lo que piensan, dicen y hacen con relación a ella. El problema se relaciona específicamente con el área de salud mental y las implicaciones sociales, familiares y escolares de la aparición de ésta en los menores se derivan de la existencia de dichos efectos nocivos.

Estudios sobre el fenómeno de la violencia expresan que tiene un origen social desencadenado por las frustraciones que produce la sociedad donde se encuentra cada individuo. Autores como Sigmund Freud, aseguran que la violencia es innata; es decir, que es la respuesta de unas fuerzas impulsivas que aparecen gracias al instinto de auto conservación. Sin embargo, existan o no las fuerzas de auto conservación se debe tener en cuenta la gran influencia de los medios de comunicación en su origen. Es un hecho aceptado por diferentes investigadores, la conclusión a la que llegó el Instituto de Salud Mental de los Estados Unidos, en el sentido de que la observación de violencia a través de los medios, y especialmente la violencia televisada engendra más violencia (2).

Los medios, así como la familia y la escuela, actúan como agentes de formación y socialización en los niños, ya que a través de ellos se transmiten creencias, valores y actitudes del hombre en la vida social. Es en la etapa infantil donde se da la adquisición de normas, valores y patrones de comportamiento, de conocimiento de la realidad, de creación de intereses, motivaciones, actitudes y opiniones (3). Si a través de los diferentes medios, se trasmite violencia, se contribuye con esto a generar y penetrar valores violentos en la sociedad y por consiguiente a reforzar una sociedad violenta.

Se han ofrecido diferentes explicaciones para clarificar el hecho de que la observación de la violencia engendra más violencia: Parece que hay procesos 
cognoscitivos, como emocionales implicados. Autores como Bandura (1-14), nos hablan de la teoría de la imitación, por la cual, las conductas observadas a través de los medios originan conductas parecidas en el espectador, y más aún en los niños, quienes son más vulnerables a los mensajes y toman por realidad lo que observan, especialmente en la pantalla chica (2). Los niños muy pequeños equiparan a la realidad cuanto ven allí (3); cuando los niños van creciendo adoptan nuevas definiciones de la realidad televisiva: creen que cualquier cosa que ven en la pantalla es autentica, más adelante creen que lo que ven en la televisión representa algo que probablemente sucede.

Diferentes estudios, especialmente con enfoque conductista, han demostrado el efecto de los medios en el comportamiento de los niños, se ha llegado a la conclusión que la observación de conductas y comportamientos violentos, afecta el comportamiento en los niños, despertando o aumentando su agresividad. Otros estudios han relacionado la observación de conductas violentas con la manifestación posterior de conductas delincuenciales. Estudios como el de Dorr (2) han demostrado que el grupo de edad al cual puede causarle más daño es el de los niños entre ocho y doce años.

Los efectos de la observación de la violencia en los medios, se pueden ver desde dos vías: los medios en muchas ocasiones incitan a la violencia y la observación de la violencia a través de éstos predispone a los individuos hacia ella (2). Quizás el efecto de la violencia en los medios más estudiado es el efecto sobre el comportamiento, el cual se manifiesta en forma de agresividad. Otros efectos de la violencia son: la insensibilidad y la modificación o creación de valores y normas. Bandura, en los años sesenta, fue quizá el pionero de la investigación sobre los efectos de la violencia en los niños. A través de diferentes investigaciones pudo constatar como los niños imitan la violencia observada. Posteriormente, buscó a través de otros estudios determinar si se imitaba el comportamiento agresivo, bien fuera que este lo ejecutara una persona o un dibujo animado. Los resultados de tales estudios mostraron que hubo tanta imitación en el grupo de los niños que vio al adulto agresivo como en el grupo donde el agresor era un dibujo animado (4).

Ross y colaboradores (5), mostraron además que los efectos pueden ser duraderos. Liebert y Poulos encontraron una relación significativa entre la exposición a películas violentas y la tendencia a comportarse de manera agresiva. Chaffé y McLeod (4) encontraron que los sujetos que veían programas violentos afirmaron aprender de estos, formas de devolver injurias y de hacer cosas sin ser sorprendidos. Parke, Berkowitz y West (5), en 1977, mostraron que la expo- 
sición a películas violentas aumentaba la conducta agresiva en el espectador. La conclusión de los estudios mencionados es que la observación de la violencia a través de los medios de comunicación produce comportamientos agresivos.

La insensibilidad frente a la violencia es otro fenómeno relacionado con el efecto de la observación de la violencia mencionado en la literatura científica. Numerosos estudios han mostrado que la observación repetida de la violencia puede llevar a las personas a habituarse a ella, lo cual puede llevar a disminuir su disposición para actuar y prestarle ayuda a las victimas y puede, además, llevar a ver la violencia como algo natural y a usarla cuando se presenten circunstancias provocadoras (4).

Los estudios de George Gerbner en los Estados Unidos, son referente obligado en el tema de la violencia en los medios, ya que crearon los índices de violencia en los programas televisivos (5). Dichas investigaciones comprobaron la correspondencia entre la violencia real y la violencia televisada, dependiendo del grupo social del televidente. A través de estos estudios, se llegó a la conclusión que los grupos de menores ingresos y los vecindarios violentos tienen predisposición a la violencia (6) y más exposición a la televisión violenta. Otros estudios han demostrado que en las personas de clase socioeconómica baja, las acciones violentas de los héroes reciben mayor aprobación y se admira más a los violentos. Estas relaciones se explican por la frustración social.

En Colombia se han realizado pocos trabajos respecto al fenómeno del efecto de la violencia observada por los niños a través de los medios de comunicación. Un trabajo, realizado con niños de Bucaramanga, mostró que los niños aprenden en la televisión el uso de la violencia para lograr sus fines (3). Además, este estudio evidenció que la programación infantil colombiana se caracteriza por la inclusión de series animadas de acción, donde la agresión y la violencia son elementos predominantes.

\section{MATERIALES Y MÉTODOS}

La presente investigación es de tipo descriptivo-exploratorio (7). Se tomó como población la totalidad de los estudiantes matriculados en los diferentes cursos de una institución educativa del sector oficial, en el periodo agosto- diciembre de 2006. Se seleccionaron mediante muestreo aleatorio estratificado 60 niños, alumnos de primero hasta quinto de primaria, pertenecientes a la Comuna 20 de la Ciudad de Cali. La población esta compuesta principalmente por los estratos socioeconómicos 1,2 y 3 (8). 
Los participantes diligenciaron dos encuestas, una dirigida a escolares entre 6 y 8 años y otra a preadolescentes entre 10 y 12 años. Mediante estas encuestas se recolectó información sobre variables sociodemográficas como: edad, sexo, nivel educativo, etnia, barrio, tipo de familia (nuclear y extensa) y ocupación de los padres. También se recolectó información sobre los factores de riesgo a los cuales estaban expuestos los menores como: consumo de alcohol, consumo de sustancias psicoactivas y consumo de cigarrillo en el grupo familiar. Mediante la última parte de las encuestas se recolectó información respecto a la opinión sobre la presencia de violencia percibida por los menores a través de los medios de comunicación, por medio de preguntas abiertas. Previa visita a la institución educativa, se solicitó a los directores y a los padres y/o representantes de los menores, la autorización para la aplicación de las encuestas, las cuales fueron elaboradas por la investigadora y sometidas a validación de expertos. La aplicación de las encuestas fue realizada por la investigadora, previa obtención del consentimiento informado así como del asentimiento del menor. Para el procesamiento y análisis estadístico de los datos se empleó el programa SPSS (9).

Para la presente investigación se tuvo en cuenta especialmente la televisión; el medio al que los niños tienen más fácil acceso y consagran un mayor número de horas, pero también se incluyó el cine, el periódico y las presentaciones como los títeres. No se tuvieron en cuenta los computadores, los video-juegos ni la radio. El estudio tuvo el aval del el Comité de Ética de la Facultad de Salud de la Universidad del Valle (10-11).

\section{RESULTADOS}

Características sociodemográficas

El $50 \%$ de los niños estudiados correspondió a niños entre 6 y 8 años, siendo la mayoría los niños de 8 años en un $63,3 \%$. El otro $50 \%$ correspondió a preadolescentes entre 10 y 12 años, con una mayoría de niños de 10 años $66,7 \%$. El promedio de edad de los participantes fue de 8.97 años, mediana 9 y moda 8 años. La edad del grupo fue prácticamente homogénea. Su coeficiente de variación no pasa de 0,2 . Otras características sociodemográficas aparecen en la Tabla 1.

Con respecto a la etnia, $56,7 \%$ eran de etnia blanca, $30 \%$ corresponde a indígenas y $13,3 \%$. de etnia negra. El $86,6 \%$ pertenecían al estrato uno, el $11,7 \%$ al estrato dos y el $1,7 \%$ al estrato tres. 
En cuanto al tipo de familia del niño, se encontró que el 46,7 \% tenían una familia extensa, el $45 \%$ familia nuclear y el 8,3\% familia extensa modificada. La mayoría de los niños tenían entre tres y siete hermanos.

Tabla 1. Distribución según edad, sexo y grado de escolaridad, de los niños estudiados en una institución oficial de la ciudad de Cali, Colombia.

\begin{tabular}{|c|c|c|}
\hline Variables & Frecuencia & $\%$ \\
\hline \multicolumn{3}{|l|}{ Edad (años) } \\
\hline 6 & 5 & 16.7 \\
\hline 7 & 6 & 20 \\
\hline $\begin{array}{l}8 \\
9\end{array}$ & 19 & 63.3 \\
\hline 10 & 20 & 66.7 \\
\hline 11 & 6 & 20 \\
\hline 12 & 4 & 13.3 \\
\hline \multicolumn{3}{|l|}{ Sexo } \\
\hline Masculino & 42 & 70 \\
\hline Femenino & 18 & 30 \\
\hline \multicolumn{3}{|l|}{ Grado } \\
\hline Primero & 6 & 10 \\
\hline Segundo & 2 & 3.3 \\
\hline Tercero & 22 & 36.7 \\
\hline Cuarto & 10 & 16.7 \\
\hline Quinto & 20 & 33.3 \\
\hline
\end{tabular}

La ocupación de los padres fue la siguiente: 78,3 \% trabajan en actividades de economía informal como vendedores, obreros, conductores de vehículos de servicio público y vigilantes. El 11,7 \% estaban desempleados.

El $50 \%$ de las madres trabajan. Las madres que trabajan se dedican a actividades como vendedoras $15 \%$, aseadoras $13,3 \%$, niñeras $5 \%$ y a otras actividades $16,7 \%$. Es importante tener en cuenta que dado que la mayoría de los padres y las madres trabajan, los niños permanecen la mayor parte del tiempo solos en casa, lo cual influye tanto en su cuidado como en su proceso de formación. Así mismo, las ocupaciones de los padres y madres son principalmente de tipo temporal, lo que repercute en la calidad de vida de las familias.

Factores de riesgo

Se identificaron como factores de riesgo a los que están expuestos los escolares y adolescentes los siguientes: $35 \%$ de los niños tenían fumadores en sus casas y $53,3 \%$ conviven con personas que consumen licor. De los menores que están expuestos a este factor de riesgo, en el 38,3\% son sus padres los que consumen licor, en el $10 \%$ son otras personas y en 3,3\% todas las personas de su hogar. En 
cuanto al consumo de sustancias psicoactivas (12), se encontró que la totalidad de los niños niegan la presencia de una persona consumidora en su hogar.

Presencia de violencia en los medios de comunicación

Los niños en edad escolar (6-8 años) la mayoría de ellos 83,3\%, ven noticieros regularmente, y $16,7 \%$ manifiestan que los noticieros no están incluidos en la programación que acostumbran a ver. De los niños que afirman ver noticias, $56,7 \%$ afirma haber visto alguna vez agresión física en ellas y $8 \%$ han visto agresión verbal.

En cuanto a los programas con dibujos animados, el $50 \%$ de los niños reportaba que han visto agresión física en estos y 13,3 \% agresión verbal. Los dibujos animados potencian la imaginación de los niños generando en ellos la creencia que la única manera de resolver los conflictos que se presenten es a través de la violencia. Estudios como el de Gerbner(5), encontraron que en las películas de dibujos animados hay tres veces más episodios violentos por hora que en las películas para adultos.

Con respecto a las novelas el 83,3\% de los niños reconoció haber presenciado agresión física en ellas y $60,7 \%$ agresión verbal. El 56,7 \% de los niños informó haber visto agresión física en las películas. De estos, el 52,2 \% no han presenciado agresión verbal en ellas y 47,8 \% la han observado. Los resultados anteriores muestran que la presencia de agresión física es mayor que la agresión verbal en los medios lo cual muestra que estos niños están gran parte del tiempo expuestos a este tipo de comportamientos y pueden llegar a ver como normal que la forma de resolver los conflictos sea por este medio.

El 56,7 \% de la población estudiada ha visto fotos violentas en los periódicos y en las revistas, Existen medios escritos de comunicación de índole amarillista que muestran este tipo de imágenes como estrategia para su venta y en los puestos de revistas son ubicadas en los lugares más llamativos.

Al preguntar a los niños por la presencia de agresión física en los títeres el $63,3 \%$ la niegan y $96,4 \%$ de los niños, manifiesta no haber visto agresión verbal en ellas. Lo anterior muestra que a pesar del avance de la tecnología, la mayoría de los niños encuestados ven esta clase de representaciones en las que de acuerdo con los resultados del estudio, aún no ha llegado la violencia. 
Las siguientes fueron algunas de las opiniones de niños con relación a la violencia en los medios. "Es malo por que pueden aprender", "Es malo, les enseña violencia a los niños", "...los que escriben eso pongan cosas menos violentas y más amorosas", "me da pesar cuando veo noticias", "No se deben ver pues uno puede aprender de eso y de grande matar a alguien", "las películas los niños no las deberían ver, si son muñequitos no importa".

Para los niños preadolescentes (10-12 años) se encontró que 76,7 \% han visto agresión física en las noticias y $23,3 \%$ no, en este grupo de niños el $50 \%$ han presenciado agresión verbal.

El $90 \%$ de los niños acepta haber visto agresión física en los dibujos animados, el $60 \%$ afirma no haber visto agresión verbal en estas series mientras que $40 \%$ manifiesta que sí.

Todos los niños afirmaron haber visto agresión física en las novelas y el 63,7 \% reportaron agresión verbal. Estos porcentajes tan altos son preocupantes puesto que las situaciones que se presentan en este tipo de programas, tienen todas las características de una situación real motivo por el cual pueden ser aprendidas eimitadas más fácilmente.

La gran mayoría de los preadolescentes $96,7 \%$ informaron haber visto agresión física y verbal en las películas, igualmente el $100 \%$ de los niños manifestaron haber visto fotos violentas en los periódicos o revistas.

La presencia de agresión física en los títeres fue reportada por el 33,3\% de los niños y de los niños que han visto obras de títeres, el $82,8 \%$ manifestaron no haber visto agresión verbal en estas representaciones, mientras que el 17,2\% manifestaron haberlas visto.

Los preadolescentes opinaron lo siguiente con relación a la violencia en los medios: "No deberían pasar estos programas tan violentos porque hay muchos niños que los ven y se pueden matar", "es malo por los sueños, produce miedo y se acuerda uno de las imágenes que vio.", "cuando veo eso en el periódico me da miedo y pienso que me voy a soñar con eso", "la gente victima de la violencia sufre mucho", "no es normal que maten a tanta gente", "no debería existir la violencia y no deberían pasarlo por tele porque otros niños están aprendiendo de esto", "Siento tristeza".

$\mathrm{Al}$ analizar las opiniones de los preadolescentes se puede deducir que ellos también ven la televisión como un medio de aprendizaje y rechazan que a través de este medio se muestren conductas violentas. Así mismo los niños reviven las 
escenas violentas vistas en noticieros y periódicos en sus sueños. Además rechazan y comprenden el sufrimiento que ocasiona y expresan sentir tristeza ante esto.

El 41,7 \% de los preadolescentes reportaron haber visto agresión física en los noticieros. Este tipo de respuestas van acorde con la sociedad en la que vivimos, la cual se ve reflejada en los medios encargados de informar a la comunidad de lo que sucede.

\section{DISCUSIÓN}

Los hallazgos de la presente investigación muestran una significativa exposición de los niños escolares y preadolescentes a la violencia a través de los medios de comunicación. Los niños reciben señales de violencia a través de: televisión, prensa y cine; lo cual de acuerdo con la revisión bibliográfica realizada indica que la exposición de los niños a situaciones de violencia a través de la televisión y otros medios de comunicación, influye en el comportamiento de los niños al favorecer las conductas violentas $(2,4,15)$.

El trabajo fuera del hogar de ambos padres conlleva a que los niños permanezcan la mayor parte del día sin la supervisión paterna o materna. Esto influye en su cuidado y formación. Henao (13), considera que esta falta de supervisión puede incidir en el desarrollo de comportamientos antisociales.

Otro hallazgo significativo importante; es que en la opinión sobre la percepción de la violencia en los medios, influyen además aspectos relacionados con la cultura y las características sociodemográficas en las que está inserto el menor. Acorde con la literatura revisada, donde se ha demostrado que los grupos de menos ingresos económicos tienen predisposición a la violencia, sumado a la mayor exposición (en relación al tiempo) a los medios de comunicación en los cuales esta predomina: periódicos amarillistas, programas de radio, cine y televisión.

Es necesario partir de tres hechos fundamentales al analizar el papel que juegan los medios de comunicación como generadores de violencia: los medios tienen gran dosis de violencia, vivimos en una sociedad violenta y las condiciones diarias de la vida para buena parte de la población son adversas y frustrantes. Pero más que el medio en si, son el contexto social y el uso de un medio lo que determina el efecto que este causa sobre el pensamiento infantil. 
Si deseamos en Colombia una sociedad tolerante y en paz, es necesario supervisar los medios, los cuales no deben constituirse en un factor de refuerzo de la violencia. Si bien es cierto que los riesgos existen, es una ilusión pretender eliminarlos con la negación de un fenómeno como es el de la violencia en los medios de comunicación, pero se hace necesario mayor investigación en el país desde diferentes campos, con el fin de utilizarlos como un potenciador para el aprendizaje y el desarrollo de los menores de edad, teniendo en cuenta que los medios de comunicación son un excelente elemento para el proceso educativo.

Es imposible desconocer que el papel de los padres en la formación de los hijos es fundamental, pero igual de importante es el papel que nos concierne como educadores y agentes de salud, para orientar a los padres respecto al uso que se debe dar a los medios de comunicación; ayudándoles a seleccionar los medios de comunicación adecuados para sus hijos, así mismo fomentando en ellos el análisis de éstos.

Desde el área de salud mental, es importante dedicar unos talleres orientados a la formación de los educadores sobre los medios de comunicación, con el fin de enseñar a través de ellos, a los niños a establecer juicios críticos sobre la realidad de lo que contemplan a través de ellos, con lo cual se contribuye a trasformar a los niños en consumidores críticos y reflexivos del mundo social que presentan los medios.

Considerando la invasión de todos los nuevos medios de comunicación, se recomienda realizar otros estudios que informen respecto a la influencia de los computadores y los video-juegos, ya que el tema es de gran importancia para profesionales de salud, educadores y padres

Agradecimientos. A los niños, padres de familia, profesores y directivas de la Institución Educativa Eustaquio Palacios. Por su participación y apoyo, para el desarrollo de la investigación.

\section{REFERENCIAS}

1. Comisión de Estudios sobre Televisión y violencia. Televisión y violencia: informe presentado al Ministerio de Comunicaciones por la Comisión de Estudios sobre televisión y violencia. Bogotá: Fondo Colombiano de Investigaciones Científicas y Proyectos especiales "Francisco José de Caldas"; 1988.

2. Pinzón P. Elementos para orientar el problema. En: Televisión y violencia: informe presentado al Ministerio de Comunicaciones por la Comisión de Estudios sobre televisión y violencia. Bogotá: Fondo Colombiano de Investigaciones Científicas y Proyectos especiales "Francisco José de caldas"; 1988. p. 17-64. 
3. Martínez H. La actividad del televidente. En: Televisión y violencia: informe presentado al Ministerio de Comunicaciones por la Comisión de Estudios sobre televisión y violencia. Bogotá: Fondo Colombiano de Investigaciones Científicas y Proyectos especiales "Francisco José de Caldas"; 1988. p. 147.

4. Villegas M. La violencia en televisión y sus efectos. En: Televisión y violencia: informe presentado al Ministerio de Comunicaciones por la Comisión de Estudios sobre televisión y violencia. Bogotá: Fondo Colombiano de Investigaciones Científicas y Proyectos especiales "Francisco José de Caldas"; 1988. p. 187-204.

5. Marks GP. Televisión y realidad social. En: El niño y los medios de comunicación. $2^{\circ}$ edición. Madrid: Ediciones Morata; 1999. p. 62-91.

6. Invertí J. Violencia y escuela. Miradas y propuestas concretas. Argentina: Piados; 2001.

7. Pólit D, Hungler B. Investigación científica en ciencias de la Salud. $6^{\text {th }}$ edición. México: Mc Graw Hill; 2000.

8. Alcaldía de Santiago de Cali. Cali en cifras. Cali: Departamento Administrativo de Planeación; 2004.

9. SPSS for windows 13.0 Chicago SPSS Inc.; 2005.

10 Resolución 008430 de 1993, por la cual se establecen las normas científicas, técnicas y administrativas para la investigación en salud. Santa fe de Bogotá: Ministerio de Salud; 1993.

11 Ley 1090 del 6 de septiembre de 2006. Por la cual se reglamenta el ejercicio de la profesión de sicología, se dicta el código deontológico y bioético y otras disposiciones. Colombia. Diario Oficial Edición № 46383. Santa fe de Bogotá; 2006.

12 Estudio Nacional de Salud Mental. Un panorama de la Salud y enfermedad mental en Colombia. Bogotá D.C: Ministerio de Protección Social; 2003.

13. Henao J. La prevención temprana de la violencia: una revisión de programas y modalidades de intervención. Univ.psicol, 2005; 4(2):161-177.

14. Bandura A, Ribes-Inesta E. Análisis of Delinquency and aggressión. New Jersey: Lawrence Erlbaum Associates, INC; 1976.

15. Pérez I, Pinzón A, González R, Sánchez J. Influencia de la televisión violenta en niños de una Escuela Pública de Bogotá, Colombia. Revista de salud Pública, Bogotá 2005; 7(1):26-38. 